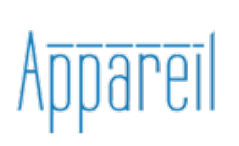

Appareil

Numéro spécial | 2008

La ville dans les sciences humaines

\title{
Diagrammes et temporalité architecturale : la loi des « appareils »
}

\section{Alexis Meier}

\section{(2) OpenEdition}

1 Journals

\section{Édition électronique}

URL : http://journals.openedition.org/appareil/332

DOI : 10.4000/appareil.332

ISSN : 2101-0714

Éditeur

MSH Paris Nord

Référence électronique

Alexis Meier, «Diagrammes et temporalité architecturale : la loi des « appareils » », Appareil [En ligne], Numéro spécial | 2008, mis en ligne le 30 juin 2008, consulté le 30 juillet 2020. URL : http:// journals.openedition.org/appareil/332 ; DOl : https://doi.org/10.4000/appareil.332

Ce document a été généré automatiquement le 30 juillet 2020

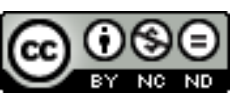

Appareil est mis à disposition selon les termes de la Licence Creative Commons Attribution - Pas d'Utilisation Commerciale - Pas de Modification 4.0 International. 


\title{
Diagrammes et temporalité architecturale : la loi des « appareils »
}

\author{
Alexis Meier
}

1 La notion d'«appareils » insiste sur le fait qu'une époque se constitue à partir de l'articulation singulière d'une loi, d'un dispositif technique et d'un support, qui tour à tour qualifie, manipule (techniquement) et enregistre, comme trace, "l'événement ». L'appareil, c'est :

ce qui met en rapport la singularité et la collectivité avec la loi, selon des normes de légitimité » [...] « La loi, c'est ce qui ouvre le matériau (le corps) à autre chose que lui : l'événement ${ }^{1}$.

2 Par exemple, le dispositif d'appareil : loi archaïque - silex - corps ou «l'urbain - le passage parisien - et la rêverie ». L'émergence visible de cette nouvelle norme renvoie à une dimension «révolutionnaire " de l'appareil en tant qu' "avènement " imprévisible d'une nouvelle temporalité : «des appareils qui articuleront donc, selon des époques différentes, selon des révolutions qu'il faudra distinguer, le corps et la loi ${ }^{2}$.» C'est l'avènement d'une nouvelle forme de rapport au présent vivant (le maintenant) qui procède par révolution (par différentiation) de la norme événementielle et donc temporelle qui définissait l'époque précédente : «Chaque appareil invente sa temporalités. » Historiquement, il n'y a donc que des époques d'appareils, étant donné que l'histoire et les faits qui en composent le matériau se donnent toujours à travers un appareillage donné de la sensibilité (qui est toujours déjà là). Il n'y a donc pas de vérité de l'histoire mais une vérité des appareils qui y découpent, fiction après fiction, l'époque :

Chaque appareil donnant son interprétation de la différence des temps, fait surgir telle ou telle temporalité qui devient son invention propre: un certain genre de fiction ${ }^{4}$.

3 La dimension esthétique de l'appareil vient du fait que pour pouvoir inscrire le nouveau mode d'accueil de l'évènement sur la surface d'inscription (Lyotard), il y a nécessairement suspension, littéralement, " une mise hors du temps » et donc une 
"fissuration " des conditions précédentes du jugement, consécutives à l'impact de l'apparition (dis-jointure) d'une œuvre sur le mode de fabrication du jugement du sujet. Il s'agit d'un « composé abstrait » mêlant résistance, révolution et réactualisation (réenregistrement) ${ }^{5}$. La dimension politique est issue du fait que la possibilité d'exercer ce jugement (divisibilité), qualifie en retour un sujet parlant qui participe à la constitution de l'être ensemble : un nouveau " partage du sensible» :

L'ensemble de l'appareil perspectif institue le spectateur quelconque en un destinataire dont le sort est réglé pour des siècles : devenir sujet. [...] Les œuvres se succèdent donc parce qu'elles mettent en œuvre l'appareil. Mais ce faisant, elles ne se bornent pas à faire reculer les bornes d'une époque de la culture, elles sont dans le même temps intériorisées par leur destinataire ${ }^{6}$.

4 L'appareil, tel un "moment du figural » marquerait un temps «immémorial » (en dehors de la surface d'inscription) qui détournerait ou bouleverserait la temporalité et induirait une inversion des rapports de la surface précédente. La condition du milieu est alors issue (par dissémination ou émission de singularités) ${ }^{7}$ d'une « révolution " des modalités du jugement provoqué par l'expérience d'une œuvre, puisqu'il s'agit à chaque fois d'une fiction inouïe qui détermine le mode d'archivage de l'événement et incidemment une nouvelle époque de l'universel par l'avènement d'une singularité. Esthétique et politique sont alors liées et mobiles, puisque le jugement issu de l'un génère la condition de l'autre. La condition de l'époque est donc la condition du milieu et comme nous pouvons l'observé chez Foucault, la production d'un milieu n'est pas neutre diagrammatiquement ${ }^{8}$. Chaque époque est «appareillée» par «son» diagramme qui détermine "le mode d'être de l'évènement». En architecture par exemple, les époques dites classiques ou modernes ont leur diagramme. Celui-ci distribue temporairement les formes et les fonctions selon un régime de visibilité spécifique (la loi). Certains diagrammes eisenmaniens, semblent quant à eux, être l'effet, par leur aspect instable, disséminé et fragmentaire, de la temporalité «postmoderne ».

5 «L'être ensemble » et la figure sont indubitablement unis par l'indécidable dérive des appareils, qui comme une sorte de différance dessinent l'époque par révolution (suspens-retournement) du rapport temps-espace-perception. Il fait "glisser» les paradigmes les uns sur les autres. À cette nouvelle temporalité correspond une nouvelle dynamique du jugement qui implique comme le précise Jean-Louis Déotte, une autre mise en forme des esprits ${ }^{9}$ :

La figure de la représentation qui s'impose amène à la présence ce qui n'était pas, ce qui n'avait pas de substance ou d'identité en lui-même : l'être ensemble ${ }^{10}$.

Pour résumer, époque après époque, on conçoit le monde par l'appareil qui lui permet à travers la médiation des œuvres, d'être lu comme tel: «Par rapport à l'appareil, les œuvres sont comme des apparitions qu'il rend possible ${ }^{11}$. On pourra alors, selon JeanLouis Déotte, «nommer », puisque celle-ci devient archivable et mémorisable, l'époque. Un sujet «nommant ", apparaîtra tel un nouvel « archiviste » enregistrant à nouveau « l'événement » sur la surface «naissante ». L'appareil, travaille avec l'irreprésentable du figural (la couleur par exemple), et ne pourra lui être «nommé ». Il s'agit d'un pur « arkhé ». Comme la Khôra platonicienne, il relève de l'immémorial et se situe comme le diagramme en dehors de l'archive et donc de l'histoire.

7 Le diagramme (l'« archi », «l'arkhé » - machine) chez certain architecte est pris comme outil de projetation architecturale a la particularité d'être un dispositif technique dont le principe est lui-même, comme nous l'avons décrit précédemment, la fabrication du 
suspens (dislocation du jugement) par décomposition (dislocation de la forme). En tant que machine à produire des traces "incalculables" la «machine» (technique) diagrammatique fabrique (tisse) chez Eisenman, un dispositif spatial inattendu, supposé ouvrir (par le bouleversement de la perception qu'il induit) à une temporalité « inouïe » (qui articule un nouveau régime de divisibilité du sensible qui souhaite faire époque). Ce diagramme (génératif) devient une sorte d'appareil de montage ou d'interface dynamique qui articule les discours et les formes, les lois et leur mode de légitimité (l'expression architecturale) selon une temporalité donnée. Instaurant « l'écart ou l'espacement (spacing) arbitraire » comme principe conceptuel et technique, ce diagramme peut être vu comme une sorte d'appareil époqual dont la temporalité serait à l'instar du montage cinématographique ${ }^{12}$ le « suspens » des autres temporalités. Comme l'architecte Peter Eisenman le précise, le diagramme agit bien au niveau de la temporalité puisqu'il permet par son principe interne de "sublimation", d'inscrire la discontinuité ${ }^{13}$ au sein du flux temporel et de rompre avec la logique causale. Comme l'appareil est un mode d'écriture de la loi sur un support par un dispositif technique, le diagramme (qui est la machine à écrire abstraite du projet) écrit la loi dans l'espace, selon un mode technique d'inscription qui est lui-même la loi des appareils. Le diagramme eisenmanien est ainsi «l'appareil» de l'époque des appareils et les bâtiments sont eux des « signes - événements » de « l'époque du suspens ».

Si le diagramme comme dispositif projectif devient une machine abstraite censée faire époque, n'y a-t-il pas risque que le dispositif (l'empreinte spatiale selon Eisenman) soit une figure, au sens littéral, de l'événement puisqu'on ne peut présenter l'imprésentable du sublime ? Si le diagramme « dis-jonctif » devient par défaut la structure abstraite du bâtiment, alors l'« archi-écriture " et la trace peuvent se confondre. Mais, à supposer qu'un dispositif spatial (un bâtiment) reste dans un suspens perpétuel de la temporalité, c'est-à-dire puisse faire évoluer le corps dans le milieu du sublime ou du figural (comme à l'intérieur d'une peinture de Barnet Newman ou de Rothko pour reprendre les exemples de Jean-Louis Déotte ${ }^{14}$, serait-il alors possible d'y enchaîner humainement? Si le diagramme produit un effet trop puissant de résistance par le rapport suprasensible ${ }^{15}$ de l'esprit sur l'objet, n'y a-t-il pas un risque de ne plus faire communauté ? Si ce travail figural ne permet plus aucun enchaînement et que la figure n'est pas régénérée mais perpétuellement "suspendue ", comme anéantie et que la "surface » reste dans l'abîme du sublime, alors les limites de cette fragmentation différentielle sont une possible non-reconstitution du sens (que serait la communauté du non-sens ?) : « Est sublime ce qui plait immédiatement par la résistance qu'il oppose à l'intérêt des sens [...] en opposition à la sensibilité ${ }^{16} »$. C'est un mirage que de penser que l'on peut «présenter» le moment «sublime» de l'appareil puisqu'il n'est pas archivable sur un support. Le «figural» doit pourtant redevenir figure pour faire communauté $^{17}$. C'est là, tout le paradoxe du diagramme et de la " presentness $^{18}$ » de Peter Eisenman qui tentent de maintenir la figure dans le figural pour avoir accès à un domaine de la «sensibilité » a-temporelle et donc perpétuellement "vierge » affectivement. L'« économie » disjonctive de la forme doit faire du bâtiment une sorte d'instantané du « moment sublime ». Mais ce dernier ne correspond pas forcément à un " effet» du sublime capable de provoquer notre "suspens». Il devient alors assez délicat pour Eisenman de faire de l'architecture pour tous, puisque le "sublime", comme l'époque ne peut pas se décréter. Autrement dit, la circulation abstraite des traces au sein des explorations diagrammatiques graphiques n'a pas le même statut que la disposition matérielle des traces, une fois matérialisées dans l'espace qui 
« impactent » le jugement. La divisibilité différentielle de la forme n'ouvrant donc pas nécessairement à une divisibilité du sensible, une œuvre " fragmentée " plastiquement n'est pas forcément génératrice de division au sens « du partage du sensible ». L'art de la « déliaison » (Déotte) n'entraîne pas toujours, comme on le constate aujourd'hui, le suspens, mais parfois plutôt un effet de métaphore du suspens en produisant un "design" déstructuré affirmant un "style» déconstruit. On trouve cette "production" déconstructiviste chez un certain nombre d'architectes célèbres des années 1990-2000, par exemple Coop Himmelblau, Frank Gehry, Michael Saee, Tom Mayne, Jacob McFarlane, Hadid,...). Autrement dit, la disjonction opérée par le diagramme ne doit pas être formelle, elle ne procède pas d'une image ou d'une seule technique. On peut noter ici que c'est une des raisons pour lesquelles la déconstruction architecturale a été critiquée lorsqu'elle a évolué vers un style «déstructuré ». Pour autant, l'avènement d'un « design », d'une « écriture » déconstructiviste démontre que l'époque de la déconstruction (architecturale) a bien eu lieu.

9 La question à laquelle finalement nous invitent à répondre Eisenman à partir de son désir de presentness architecturale, est de savoir à quelles conditions de la dis-jointure spatiale est-il pensable d'obtenir au-delà de la différence "formelle", un effet « suspens » qui ouvre à la "révolution ». À quelles conditions de l'Informe obtient-on l'effet de la presentness? Selon Derrida, une « interruption, rupture de l'enchaînement habituel du temps ${ }^{19}$ " bouleverse la structure relationnelle du milieu et "ouvre à la survenue de l'évènement comme autre ${ }^{20}$ », une "autre » époque. Entre conscience et matière, une question irrésolue semble poser une limite à la démarche diagrammatique d'Eisenman: à quelle condition, la différence spatiale produit-elle la différence du politique? Ce problème délicat concerne directement la relation du diagramme aux signes matériels (de l'archi-trace à la trace ou à l'empreinte dirait Eisenman ${ }^{21}$ ) puis des signes à leur perception. Andrew Benjamin a posé récemment ces questions en partant de l'Informe chez George Bataille :

l'Informe doit être relié à une pratique architecturale [...] traduisant le déclasser de Bataille qui correspond plus ou moins à la destabilisation chez Eisenman [...] Tous ces termes posent le même problème, ils doivent être présent dans et par la réalisation du bâtiment comme bâtiment d'architecture ${ }^{22}$.

La réponse à cette question qui rapproche les conditions de la matière aux conditions de l'aperception et donc du jugement, semble nous inviter à une phénoménologie de la différence. 


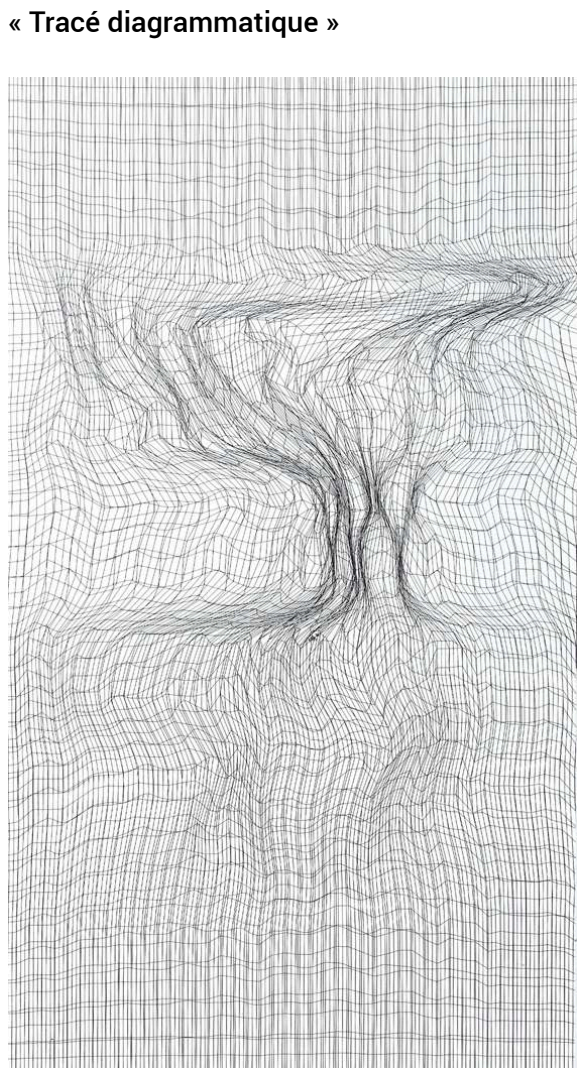

Peter Eisenman

11 Peter Eisenman, "Tracé diagrammatique » collectant et mélangeant l'enregistrement d'une d'activité neuro-synaptique à différentes fréquences (donnant un principe spécifique de distribution de traces) et la topographie du site (les traces), afin de déterminer le volume bâtit, pour la Bibliothèque IUHEI, place des Nations, Genève, 1997.

\section{BIBLIOGRAPHIE}

Benjamin Andrew, « Opening the Interstitial: Eisenman space of difference », "Blurred Zones: Investigations of the Interstitial”: Eisenman Architects 1988-1998, New-York, Monacelli Press, 2003.

Déotte Jean-Louis, L'époque des appareils, document de travail du séminaire doctoral, 2004.

Eisenman Peter, « The diagram and the becoming unmotivated of the sign », Diagram Diaries, Londres, Thames and Hudson, 1999.

Foucault Michel, Surveiller et Punir, Paris, Gallimard, 1975.

Eisenman Peter, « Tracer Eisenman - Entretien avec Alexis Meier », Archistorm, n ${ }^{\circ \circ} 20,2006$, p. $40-43$.

Kant Emmanuel, Critique de la faculté de juger, Paris, Gallimard (Folio), 1995. 
Manesse Laurence, « Le figural comme milieu », in Appareil et intermédialité, Paris, L’Harmattan, 2007.

Rousse Pascal, « L'Architectonique du montage selon Eisenstein et Benjamin : architecture temporelle et transformation du lieu », Revue en ligne www.cadrage.net, octobre 2006.

\section{NOTES}

1. Jean-Louis Déotte, L'époque des appareils, document de travail du séminaire doctoral, 2004, p. 12.

2. Ibid., p. 2.

3. Jean-Louis Déotte, « Le Milieu du monde », 2005.

4. Ibid., p. 10.

5. «Les révolutions des appareils, alors c'est le mode d'accueil de l'événement qui change. L'événement, c'est par définition l'imprévisible. Ce qui implique qu'un mode d'accueil esquisse, prédétermine, ce qui sera pour lui événement ou non : s'ouvrant à telle ou telle part d'imprévu, la constituant comme monde. ", Jean-Louis Déotte, in L'époque des appareils, p. 17.

6. Ibid., p. 20-21.

7. «Chaque appareil est donc partagé entre un devenir programmatique (le dispositif) et une déconstruction systématique. Les deux ne se recouvrent pas, car la déconstruction artistique trouve son énergie d'une manière originale. Elle est comme tirée en avant par une énigme : celle de l'irruption imprévisible de l'appareil comme ce qui a fait époque. Le moment de l'irruption de l'appareil qui n'est pas celui de l'invention technique. Mais l'instant où pour un certain public, il est devenu évident, que du fait de tel appareil «on » changeait d'époque [...] C'est le mode d'accueil de l'événement qui change. L'événement, c'est par définition l'imprévisible. Ce qui implique qu'un mode d'accueil esquisse, prédétermine ce qui sera pour lui événement ou non: s'ouvrant à telle ou telle part d'imprévu, la constituant comme monde. ", Jean-Louis Déotte, in L'époque des appareils, p. 16.

8. Voir l'analyse de Foucault sur le Panoptique, «Le Panoptique [...] c'est le diagramme d'un mécanisme de pouvoir ». Michel Foucault, Surveiller et Punir, Paris, Gallimard, 1975, p. 207.

9. Ibid., p. 21.

10. Jean-Louis Déotte, « Le Milieu du monde », 2005.

11. Ibid., p. 18.

12. "Le montage est donc construit sur un rapport du temps à l'espace architectural "concret" tel que nous le reconstruisons mentalement et à l'espace architectural "abstrait", architectonique - celui que le film présente et dont nous faisons l'expérience en une image conceptuelle (obraz) - de l'escalier filmique comme contexte, fond mouvant, tectonique, des figures et de l'action, dé-mesure quantitative et qualitative de leurs positions respectives et, enfin, index du montage lui-même comme architecture temporelle.» Pascal Rousse, "L'Architectonique du montage selon Eisenstein et Benjamin: architecture temporelle et transformation du lieu », Revue en ligne, www.cadrage.net, octobre 2006.

13. «Mais il y a aussi une temporalité impliquée dans le processus du diagramme. Derrida dit que le Bloc Magique inclut dans sa structure ce que Kant décrit comme les trois modes du temps: permanence, succession, simultanéité. Le diagramme, comme le Bloc Magique, contient la simultanéité de l'apparence sur sa surface, ce qui serait apparenté aux lignes noires sur la couche $\mathrm{du}$ dessus, aussi bien que l'indentation dans la cire en dessous; Le second aspect du temps du diagramme est la succession, qui est apparenté au soulèvement de la tablette et son implication sur l'effacement et l'affichage d'une nouvelle image. Puis la permanence dans la cire elle-même. Le diagramme présente dans un tel contexte une conception discontinue du temps dans la périodicité et l'espacement de l'écriture. Ces trois conditions du temps ne sont pas linéaires ou 
connectées de manière narrative. Alors, le diagramme est une condition intermédiaire ou interstitielle qui s'étend entre temps et espace, entre l'objet architectural et l'intériorité de l'architecture. ", Peter Eisenman, "The diagram and the becoming unmotivated of the sign ", Diagram Diaries, Londres, Thames and Hudson, 1999, p. 34.

14. "L'art à l'époque de la disparition, qui s'expose à Santiago ou à Buenos Aires par exemple, n'appartient plus à la catégorie du sublime esthétique comme pouvait le faire l'abstraction d'un B. Newman ou d'un Rothko ». Jean-Louis Déotte, in L'époque des appareils, document de travail du séminaire doctoral, 2004, p. 24.

15. «Le sublime réside simplement dans la relation où ce qui est de l'ordre du sensible dans la représentation de la nature est jugé susceptible d'être utilisé au niveau suprasensible " Kant, "Analytique du beau », chapitre 16, Critique de la faculté de juger, Paris, Gallimard (Folio), 1995, p. 210.

16. Ibid., p. 211.

17. « On n'enchaîne pas sur le sublime », Laurence Manesse, «Le figural comme milieu », Appareil et intermédialité, Paris, L'Harmattan, 2007, p. 158.

18. Peter Eisenman témoigne en 2002 de sa volonté d'exploiter dans son architecture de cette « sensibilité non émotionnelle » du temps dans l'espace :

Alexis Meier : c'est ce que vous appelez la presentness?

Peter Eisenman: La presentness est la capacité de la dimension affective de l'architecture de rester affective à travers le temps. En d'autres termes, de ne pas devenir instinctuelle, par exemple: si vous allez à Venise, sur le pont Rialto, le pont Rialto n'a plus de dimension affective [...] Vous prenez une photo, vous la regardez, c'est joli, mais ça n'a aucune dimension affective. La presentness est la capacité de quelque chose comme le Rendetorre de Palladio de maintenir sa dimension affective à travers le temps. Par exemple: à mon sens, la chapelle de Ronchamp est devenue instinctuelle. Le couvent Tourette comporte la presentness parce qu'il reste affectif. Et personne n'est encore arrivé à ramener La Tourette à une sorte de normalité de situation, ce qui n'est pas le cas de Ronchamp.

"Peter Eisenman, L'architectonique de l'accident, entretien avec Alexis Meier», in AppareilAccident, revue en ligne www.criticalsecret.com, théma - anachronique - Double numéro ${ }^{\text {oso }} 11-12$, été-automne 2003.

19. Jacques Derrida, Émission radiophonique, Du jour au lendemain, France Culture, décembre 1993.

20. Ibid.

21. «Ce que nous entendons le plus souvent par l'idée de trace en architecture, c'est en fait l'empreinte. », Peter Eisenman, in « Tracer Eisenman - Entretien avec Alexis Meier », Archistorm, $\mathrm{n}^{\circ \circ} 20,2006$, p. $40-43$.

22. Andrew Benjamin, "Opening the Interstitial: Eisenman space of difference", "Blurred Zones: Investigations of the Interstitial": Eisenman Architects 1988-1998, New-York, Monacelli Press, 2003, p. 307.

\section{RÉSUMÉS}

La notion d'«appareils» insiste sur le fait qu'une époque se constitue à partir de l'articulation singulière d'une loi, d'un dispositif technique et d'un support, qui tour à tour qualifie, manipule (techniquement) et enregistre, comme trace "l'événement». Le diagramme semble lui, être le 
nom d'un mécanisme abstrait à partir duquel l'espace et le temps peuvent indéfiniment se différentier et «former » ainsi les surfaces «épokales ». En partant de la démarche déconstructiviste de l'architecte américain Peter Eisenman, nous proposons d'identifier, à partir de leur mécanisme réciproque, les interrelations possibles entre la notion de diagramme et d'appareil.

INDEX

Mots-clés : algorithme, appareil, autonomie, conscience, décomposition, diagramme, différance, différence, dispositif, écriture, environnement, espace, éthique, événement, expérience, forme, génétique, index, milieu, politique, signe, singularité, structure, texte, trace

\section{AUTEUR}

ALEXIS MEIER

Architecte, Docteur en philosophie 